\title{
Raman-induced Kerr effect microscopy with balanced detection ${ }^{\dagger}$
}

\section{Egle Molotokaite, Vikas Kumar, Cristian Manzoni, Dario Polli, Giulio Cerullo* and Marco Marangoni}

\begin{abstract}
We present a new coherent Raman scattering technique, which we call balanced-detection Raman-induced Kerr effect. The technique relies on a balanced detection architecture, inspired to that applied for electro-optic sampling in the terahertz domain, which allows one to sensitively measure the Raman-induced Kerr effect-induced polarization rotation of the Stokes field. Balanced detection allows for an intrinsic rejection of laser noise, thus making possible to approach shot-noise limited conditions even when using fiber laser sources and relatively low modulation frequencies. Balanced-detection Raman-induced Kerr effect removes both linear and nonlinear background, provides self-heterodyne amplification of the nonlinear Raman signal, and scales linearly the sample concentration. Furthermore, by properly changing the detection conditions, it allows the reconstruction of the full complex vibrational response, both in amplitude and phase, greatly increasing chemical selectivity in single-color excitation. Copyright $\odot 2013$ John Wiley \& Sons, Ltd.
\end{abstract}

Keywords: coherent Raman spectroscopy; Raman-induced Kerr effect; balanced detection; self-heterodyning; femtosecond fiber lasers

\section{Introduction}

Coherent Raman Scattering (CRS) microscopy is gaining a growing recognition in biomedical optics ${ }^{[1]}$ thanks to its capability of noninvasive, label-free imaging of tissues and cells, ${ }^{[2]}$ exploiting the intrinsic contrast mechanism provided by the characteristic molecular vibrations of the target molecules. CRS techniques exploit third-order nonlinear optical processes ${ }^{[3]}$ that make use of two synchronized trains of laser pulses at frequencies $\omega_{p}$ (Pump frequency) and $\omega_{S}$ (Stokes frequency). When the difference between Pump and Stokes frequencies matches a characteristic vibrational frequency $\Omega$, i.e. $\omega_{\mathrm{p}}-\omega_{\mathrm{s}}=\Omega$, the molecules in the focal volume are made to vibrate in phase, setting up a vibrational coherence that can be read out in different ways depending on the adopted CRS technique. With respect to spontaneous Raman scattering microscopy, ${ }_{1}^{[4]}$ the advantages of a coherent excitation lie in a strongly enhanced signal and in the three-dimensional sectioning capabilities characteristic of nonlinear microscopy.

Until recently, the most popular form of CRS has been coherent anti-Stokes Raman scattering (CARS) ${ }_{1}^{[5]}$ in which the vibrational coherence is read out by a further interaction with the pump field, generating a coherent radiation at the anti-Stokes frequency, $\omega_{\mathrm{as}}=2 \omega_{\mathrm{p}}-\omega_{\mathrm{s}}$. CARS has the important advantage of generating a background-free signal, because it is at a different frequency with respect to pump and Stokes; this allows very high acquisition speeds, up to the video rate. ${ }^{[6]}$ The main drawback of CARS is the presence of a nonresonant background (NRB) that is generated both by the molecular species under study and by the surrounding medium, according to a four-wave mixing scheme, with the absorption of two photons at $\omega_{\mathrm{p}}$ and the simultaneous emission of one photon at $\omega_{\mathrm{s}}$ and one photon at $\omega_{\mathrm{as}}$. The NRB does not carry any chemically specific information and, when the concentrations of the target molecules are low, can distort and even overwhelm the resonant signal of interest. Several techniques have been proposed and experimentally demonstrated to suppress the NRB in CARS, such as interferometric $\mathrm{CARS}_{1}{ }^{[7,8]}$ frequency-modulation $\mathrm{CARS}^{[9]}$ polarization $\mathrm{CARS}^{\left[{ }^{[10]}\right.}$ and timedelayed $\mathrm{CARS}^{[11-13]}$ but they have not found widespread application because they significantly complicate the experimental setup. In addition, even when the NRB is negligible, the CARS signal scales as $N^{2}, N$ being the volume concentration of the targeted oscillators, so that it fails to detect the less abundant molecular species and makes their quantitative assignment difficult.

These limitations of CARS can be overcome by the use of a different CRS technique, stimulated Raman scattering (SRS). ${ }^{[14,15]}$ In SRS, the coherent interaction with the sample induces stimulated emission from a virtual state to the investigated vibrational state of the molecule, resulting in a Stokes-field amplification (stimulated Raman gain) and in a simultaneous pump-field attenuation (stimulated Raman loss). SRS is inherently free from NRB, and in addition, it scales linearly with molecular concentration, allowing the quantitative detection of even diluted species. ${ }^{[16]}$ On the other hand, SRS requires the measurement of a weak differential transmission signal (stimulated Raman gain or stimulated Raman loss) sitting on top of the large (and noisy) linear background given by the Stokes (pump) light. Extraction of such signal requires modulation of the pump (Stokes) light and synchronous detection. To reach video-rate imaging, the detection chain needs

\footnotetext{
* Correspondence to: Giulio Cerullo, IFN-CNR, Dipartimento di Fisica, Politecnico di Milano, Piazza Leonardo da Vinci 32, 20133 Milano, Italy E-mail: giulio. cerullo@fisi.polimi.it

+ This article is from the ECONOS part of the joint special issue on the European Conference on Nonlinear Optical Spectroscopy (ECONOS 2012) with Guest Editors Johannes Kiefer and Peter Radi and the II Congresso Nationale di Spettroscopie Raman ed Effetti Ottici Non Lineari (GISR 2012) with Guest Editor Maria Grazia Giorgini.
}

IFN-CNR, Dipartimento di Fisica, Politecnico di Milano, Piazza Leonardo da Vinci 32, 20133 Milano, Italy 
to work under shot-noise-limited conditions at high acquisition speeds, which is possible only at modulation frequencies in excess of a few megahertz. ${ }^{[17,18]}$ So far, shot-noise limited performance in SRS has been achieved using stable solid state laser systems, such as synchronized Ti:sapphire lasers ${ }^{[19]}$ or optical parametric oscillators pumped by $\mathrm{Nd}: \mathrm{YVO}_{4}$ oscillators, ${ }^{[20]}$ which present particularly favorable noise properties at high frequencies. Such systems are however complex, expensive, and with a large footprint. When using the more compact, versatile, and cost-effective fiber lasers, their poorer noise performance can prevent the signal-to-noise ratio from reaching the shot-noise limit. ${ }^{[21,22]}$ This problem can be overcome by employing balanced detection schemes, as recently reported by Nose et al. ${ }^{[22]}$ and Gambetta et al. ${ }^{[23]}$. The technical difficulties associated with signal detection have so far limited the diffusion of SRS in mainstream biomedical imaging. ${ }^{[24]}$

A well-known but less explored CRS technique is Ramaninduced Kerr effect (RIKE). ${ }^{[25]}$ RIKE relies on the Raman-induced birefringence that occurs when the pump-Stokes frequency detuning is in resonance with a vibrational transition, leading to a modification of the polarization state of the Stokes(pump) field. Such change is typically detected by placing a crossed polarizer after the sample, blocking the original Stokes(pump) field while transmitting the orthogonally polarized nonlinear field component generated by RIKE, allowing one to acquire a signal free of linear background as in CARS. When using circularly polarized pump(Stokes) pulses, it is in addition possible to suppress the NRB. ${ }^{[26,27]}$ Such advantages are traded off by a rather poor signal and by the quadratic dependence of the RIKE signal on the sample concentration. In a different configuration,

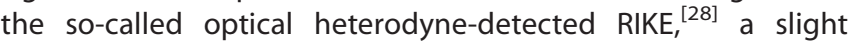
detuning of the crossed polarizer allows the transmitted field to act as a local oscillator (LO) that interferes with the RIKE field component, resulting in an optical heterodyne amplification of the nonlinear signal and in a linear concentration dependence. This happens, however, at the expense of the linear background, which increases proportionally to the amount of angular detuning of the polarizer. In addition, the optical heterodyne-detected RIKE signal is superimposed with the SRS signal of the LO, thus limiting the maximum intensity of the LO itself. ${ }^{[28]}$

In this paper, we present a new CRS technique, which we call balanced-detection RIKE (BD-RIKE). We demonstrate, both theoretically and experimentally, that a balanced detection architecture, inspired to that applied for electro-optic sampling in the terahertz domain, ${ }^{[29,30]}$ allows one to sensitively measure the RIKE-induced polarization rotation of the Stokes (pump) field. Balanced detection allows for an intrinsic rejection of laser noise, thus making possible to approach shot-noise limited conditions even when using fiber laser sources and relatively low modulation frequencies. When compared with more common CRS approaches such as CARS or SRS, BD-RIKE offers a series of advantages: (i) the absence of any linear or nonlinear background, and thus of any spectral distortion with respect to the spontaneous Raman response of the sample, (ii) the self-heterodyne amplification of the nonlinear Raman signal, and (iii) the linear dependence of the signal on the sample concentration. Furthermore, we will show that BD-RIKE offers the possibility to measure both the real and the imaginary part of the nonlinear susceptibility, thus allowing one to reconstruct the full complex vibrational response, both in amplitude and phase. The availability of the complex vibrational response increases the chemical selectivity, enabling to distinguish, even with single-color excitation, different molecular constituents with overlapping Raman spectra.

\section{Theoretical considerations}

Comparison of different coherent Raman spectroscopy techniques

To emphasize the merits of our BD-RIKE approach, in the following, we will review and compare the performances of different CRS techniques. In general, a CRS process can be described as a fourwave mixing interaction mediated by the third-order nonlinear susceptibility of the medium. ${ }^{[3]}$ The interaction mixes four fields at frequencies $\omega_{i}$, where $i=1, \ldots, 4$, with the condition $\omega_{1}-\omega_{2}+\omega_{3}=$ $\omega_{4}$. Without loss of generality, the nonlinear signal can be thought as generated at the frequency $\omega_{4}$ according to the equation:

$$
\frac{\partial E_{4}}{\partial z}=-i \alpha_{4} P_{4}^{(3)} \exp (i \Delta k z)
$$

where $z$ is the propagation direction, $P_{4}^{(3)}=\varepsilon_{0} \chi^{(3)} E_{1} E_{2}^{*} E_{3}$ is the third-order nonlinear polarization, $\Delta k=k_{1}-k_{2}+k_{3}-k_{4}$ is the wave-vector mismatch and $\alpha_{4}=\frac{3 \omega_{4}}{4 c n_{4}}, c$ being the speed of light and $n_{4}$ the refractive index of the medium at $\omega_{4}$. The nonlinear susceptibility $\chi^{(3)}$ of the sample can be decomposed in two terms as according to the equation:

$$
\chi^{(3)}=\chi_{N R}^{(3)}+\chi_{R}^{(3)}=\chi_{N R}^{(3)}+\operatorname{Re}\left[\chi_{R}^{(3)}\right]+\operatorname{Im}\left[\chi_{R}^{(3)}\right]
$$

where $\chi_{R}^{(3)}$ is the resonant nonlinear susceptibility given by the molecules under study and $\chi_{N R}^{(3)}$ is the NRB generated both by the molecules and by the surrounding medium. Note that, although $\chi_{N R}^{(3)}$ is real and frequency independent, $\chi_{R}^{(3)}$ is the superposition of several complex Lorentzian responses related to the different vibrational transitions of the molecule:

$$
\chi_{R}^{(3)}=\sum_{i} \frac{N_{i} \sigma_{i}}{\omega_{1}-\omega_{2}-\Omega_{i}-i \Gamma_{i}}
$$

where $N_{i}$ is the concentration of Raman active scatterers, $\sigma_{i}$ is the cross-section, $\Omega_{i}$ is the resonance frequency, and $\Gamma_{i}$ is the line-width of the vibrational transition.

Let us first consider a CARS process where all interacting fields have parallel polarization along the $x$-axis. In this case, we have $\omega_{1}=\omega_{3}=\omega_{p}, \omega_{2}=\omega_{s}, \omega_{4}=\omega_{a s}$ and $\chi^{(3)}=\chi_{x x x x}^{(3)}$. By solving Eqn (1) in the small-signal limit under phase-matched conditions $(\Delta k \cong 0)$, the nonlinear signal becomes the following:

$$
E_{a s}=-i \alpha_{a s} \chi^{(3)} E_{p}^{2} E_{s}^{*} L
$$

where $L$ is the nonlinear interaction length within the Ramanactive medium. Because the CARS field is at a different frequency with respect to pump and Stokes, it can be easily measured in a background-free manner by directly detecting its intensity (Fig. 1(a)):

$$
\begin{gathered}
S_{C A R S}\left(\omega_{p}-\omega_{S}\right) \propto\left|E_{a S}\right|^{2} \propto\left|\chi^{(3)}\right|^{2} I_{p}^{2} I_{S} L^{2}= \\
=\left[\left|\chi_{R}^{(3)}\left(\omega_{p}-\omega_{S}\right)\right|^{2}+\chi_{N R}^{(3) 2}+2 \chi_{N R}^{(3)} \operatorname{Re}\left[\chi_{R}^{(3)}\left(\omega_{p}-\omega_{S}\right)\right]\right] I_{p}^{2} I_{S} L^{2}
\end{gathered}
$$

Equation (5) shows that the NRB affects considerably the CARS response, because it is responsible of a constant, frequencyindependent term $\left(\chi_{N R}^{(3) 2}\right)$ and of a dispersive interference term $2 \chi_{N R}^{(3)} \operatorname{Re}\left[\chi_{R}^{(3)}\left(\omega_{p}-\omega_{S}\right)\right]$ that distorts the Lorentzian-shaped vibrational lines. In addition, even when the NRB is negligible $\left(\chi_{N R}^{(3)} \ll \chi_{R}^{(3)}\right)$, the CARS signal scales quadratically with the 

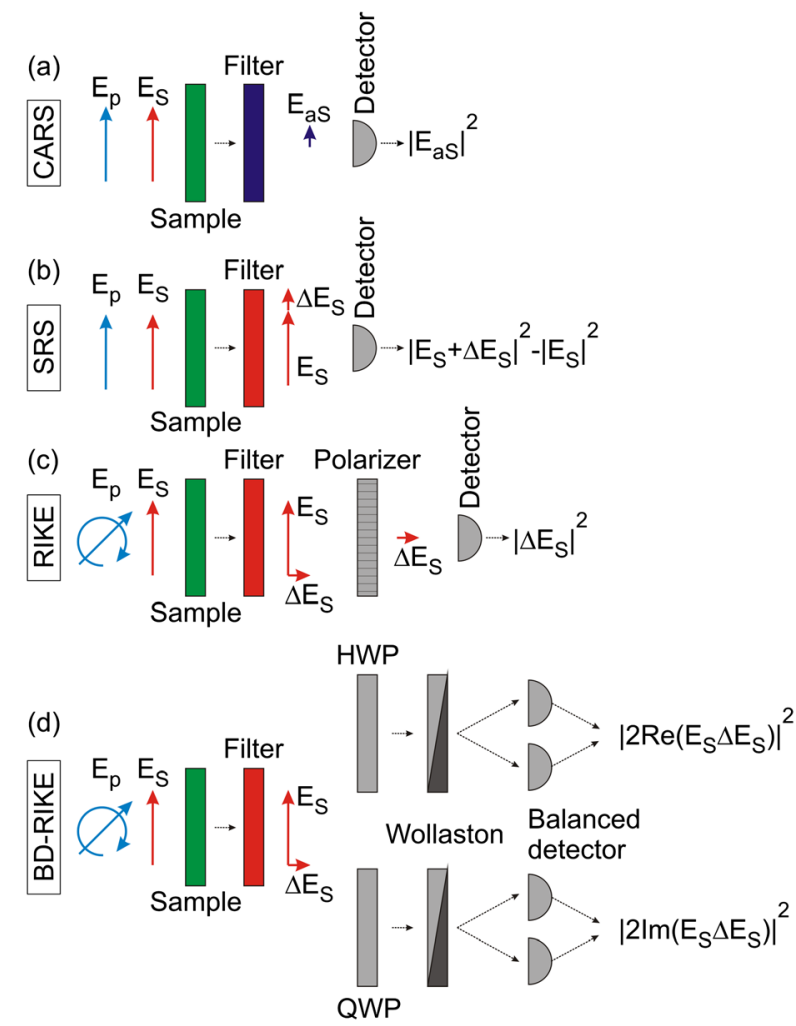

Figure 1. Sketch of the polarization states and the main optical elements for the four coherent Raman techniques described in the text (CARS, SRS, RIKE, and BD-RIKE). $E_{p}, E_{s}$, and $E_{a s}$ denote the pump, Stokes, and anti-Stokes electric fields, respectively. The polarization states are drawn in the plane perpendicular to the beam direction for clarity. HWP, half-wave plate; QWP, quarter-wave plate; CARS, coherent antiStokes Raman scattering; SRS, stimulated Raman scattering; RIKE, Raman-induced Kerr effect; BD-RIKE, balanced-detection RIKE. This figure is available in colour online at wileyonlinelibrary.com/journal/jrs.

concentration $N$, thus complicating a quantitative determination of the signal and preventing the measurement of highly diluted species.

These drawbacks of the CARS approach can be overcome by SRS, for which we have $\omega_{1}=\omega_{2}=\omega_{p}, \omega_{3}=\omega_{4}=\omega_{S}$ and again $\chi^{(3)}=\chi_{x x x x}^{(3)}$ because all the fields have parallel polarizations. In the weak signal limit, the nonlinear signal at the sample output upon integration of Eqn (1) can be written as follows:

$$
\Delta E_{S}=-i \alpha_{s} \chi^{(3)}\left|E_{p}\right|^{2} E_{S} L
$$

Because $\Delta E_{S}$ sits on top of the very large Stokes signal $E_{S}$, its extraction needs the application of a high-frequency modulation transfer technique from the pump beam. Assuming $\left|\Delta E_{S}\right| \ll E_{S}$, the measured SRS signal thus becomes(Fig. 1(b)) as follows:

$$
S_{\mathrm{SRS}} \propto\left|E_{S}+\Delta E_{S}\right|^{2}-\left|E_{S}\right|^{2} \cong 2 E_{S} \operatorname{Re}\left[\Delta E_{S}\right] \propto \alpha_{S} \operatorname{Im}\left[\chi^{(3)}\right] I_{p} I_{S} L
$$

that is proportional to the imaginary part of the Lorentzianshaped nonlinear susceptibility, without any spurious contribution from the NRB, which is effectively suppressed being a real quantity. In addition, the SRS signal is directly proportional to $\chi^{(3)}$, so that it scales linearly with the sample concentration, allowing for an easier quantitative detection. Finally, one should notice that the small nonlinear signal $\Delta E_{S}$, which is of the same order as $E_{a s}$, is multiplied by the large and phase-locked Stokes field $E_{S}$ (self-heterodyning configuration), allowing for a large amplification of the SRS signal $\left(\approx 2 E_{S} \Delta E_{S}\right)$ with respect to CARS $\left(\approx E_{a s}^{2}\right)$.

For RIKE, similar to SRS, we have $\omega_{1}=\omega_{2}=\omega_{p}, \omega_{3}=\omega_{4}=\omega_{S}$, but the polarization states of pump and Stokes pulses are different. Depending on whether the polarization rotation of the pump or of the Stokes beam is measured, four different polarization schemes are possible. For the sake of simplicity, however, we will limit hereafter the discussion to the case of a Stokes' detection only, with the pump field being either linearly polarized at $45^{\circ}$ with respect to the Stokes, or circularly polarized according to the schemes of Fig. 1. In the first case, the pump beam path, i.e. the excitation path, is equipped with a half-wave plate (HE configuration), and the corresponding effective nonlinear susceptibility is given by the following: ${ }^{[31]}$

$$
\chi^{(3)}=\chi_{x x y y}^{(3)}+\chi_{x y x y}^{(3)}
$$

The second case requires a quarter-wave plate on excitation (QE configuration) and involves an effective nonlinearity of the form: ${ }^{[31]}$

$$
\chi^{(3)}=i\left[\chi_{x x y y}^{(3)}-\chi_{x y x y}^{(3)}\right]
$$

Whatever configuration is applied, the nonlinear RIKE signal is generated with a $y$ polarization, perpendicular to that of the impinging Stokes:

$$
\Delta E_{S} \propto-i \chi^{(3)}\left|E_{p}\right|^{2} E_{S} L
$$

The typical way to detect $\Delta E_{S}$ makes use of a crossed polarizer (see sketch in Fig. 1(c)) followed by a detector that is sensitive to the intensity of the nonlinear signal. This results in an expression for the RIKE signal:

$$
S_{\mathrm{RIKE}} \propto\left|\Delta E_{S}\right|^{2} \propto\left|\chi^{(3)}\right|^{2} I_{p}^{2} I_{S} L^{2}
$$

that is similar to that for CARS, but with an important difference in the case of QE excitation. In such case, the NRB vanishes due to the fact that, according to Kleinmann symmetries, ${ }^{[31]} \chi_{x x y y, N R}^{(3)}=\chi_{x y x y, N R}^{(3)}$. A disadvantage, however, remains, which is the quadratic dependence on concentration.

\section{Balanced-detection Raman-induced Kerr effect}

Balanced-detection RIKE relies on a balanced detection technique, that is commonly used to sensitively measure polarization rotations induced by, e.g. a strong terahertz field. ${ }^{[29,30]}$ Its experimental configuration is shown in Fig. 1(d) and consists of three elements: a birefringent plate, a Wollaston prism, and a pair of identical photodiodes followed by a differential amplifier. The birefringent plate can be either a half-wave plate (HWP), which rotates the input polarization by $45^{\circ}$ (optical axis forming an angle $\theta=\pi / 8$ with the $x$-axis), or a quarter-wave plate (QWP), which produces a circular polarization (optical axis forming an angle $\theta=\pi / 4$ with the $x$-axis). In both cases, the Wollaston prism splits the beam into two orthogonally polarized beams, which have equal intensity in the absence of the nonlinear signal.

The action of the balanced detection setup can be easily rationalized with the help of Jones calculus. ${ }^{[32]}$ The Jones vector of the incoming beam can be written as follows: 


$$
E^{\mathrm{IN}}=\left|\begin{array}{c}
E_{x}^{\mathrm{IN}} \\
E_{y}^{\mathrm{IN}}
\end{array}\right|
$$

where $E_{x}^{\mathbb{N}}$ is the input Stokes field (assumed real) and $E_{y}^{\mathbb{I N}}$ is the complex field component generated by the RIKE effect.

In the case of detection with an HWP, its Jones matrix is the following:

$$
M_{\mathrm{HWP}}=\left|\begin{array}{cc}
\cos 2 \theta & \sin 2 \theta \\
\sin 2 \theta & -\cos 2 \theta
\end{array}\right|
$$

which, for the adopted HWP orientation $(\theta=\pi / 8)$, becomes the following:

$$
M_{\mathrm{HWP}}=\frac{1}{\sqrt{2}}\left|\begin{array}{cc}
1 & 1 \\
1 & -1
\end{array}\right|
$$

The output beam then becomes the following:

$$
E^{\mathrm{OUT}}=M_{\mathrm{HWP}} E^{\mathrm{IN}}=\frac{1}{\sqrt{2}}\left|\begin{array}{l}
E_{x}^{\mathrm{IN}}+E_{y}^{\mathrm{IN}} \\
E_{x}^{\mathrm{IN}}-E_{y}^{\mathrm{IN}}
\end{array}\right|
$$

The Wollaston prism separates the output beam into two orthogonal polarizations, whose intensities are measured by the two photodiodes:

$$
\begin{aligned}
& I_{x}^{\text {OUT }}=\left|E_{x}^{\mathrm{OUT}}\right|^{2}=\frac{1}{2}\left[\left|E_{x}^{\mathrm{IN}}\right|^{2}+\left|E_{y}^{\mathrm{IN}}\right|^{2}+2 \operatorname{Re}\left(E_{x}^{\mathrm{IN}} E_{y}^{\mathrm{IN} *}\right)\right] \\
& I_{y}^{\text {OUT }}=\left|E_{y}^{\mathrm{OUT}}\right|^{2}=\frac{1}{2}\left[\left|E_{x}^{\mathrm{IN}}\right|^{2}+\left|E_{y}^{\mathrm{IN}}\right|^{2}-2 \operatorname{Re}\left(E_{x}^{\mathrm{IN}} E_{y}^{\mathrm{IN} *}\right)\right]
\end{aligned}
$$

so that the signal retrieved by the balanced detector is the following:

$$
S_{\mathrm{HWP}}=I_{x}^{\text {OUT }}-I_{y}^{\mathrm{OUT}}=2 \operatorname{Re}\left(E_{x}^{\mathrm{IN}} E_{y}^{\mathrm{IN} *}\right)
$$

In the case of detection with a QWP, its Jones matrix is the following:

$$
M_{\mathrm{QWP}}=\frac{1}{\sqrt{2}}\left|\begin{array}{cc}
1+i \cos 2 \theta & i \sin 2 \theta \\
i \sin 2 \theta & 1-i \cos 2 \theta
\end{array}\right|
$$

which, for the adopted QWP orientation $(\theta=\pi / 4)$, becomes the following:

$$
M_{\mathrm{QWP}}=\frac{1}{\sqrt{2}}\left|\begin{array}{ll}
1 & i \\
i & 1
\end{array}\right|
$$

The output beam then becomes the following:

$$
E^{\mathrm{OUT}}=M_{\mathrm{QWP}} E^{\mathrm{IN}}=\frac{1}{\sqrt{2}}\left|\begin{array}{l}
E_{x}^{\mathrm{IN}}+i E_{y}^{\mathrm{IN}} \\
i E_{x}^{\mathrm{IN}}+E_{y}^{\mathrm{IN}}
\end{array}\right|
$$

and the intensities of the two orthogonal polarizations are the following:

$$
\begin{aligned}
& I_{x}^{\text {OUT }}=\left|E_{x}^{\mathrm{OUT}}\right|^{2}=\frac{1}{2}\left[\left|E_{x}^{\mathrm{IN}}\right|^{2}+\left|E_{y}^{\mathrm{IN}}\right|^{2}+2 \operatorname{Re}\left(E_{x}^{\mathrm{IN}}\left(i E_{y}^{\mathrm{IN}}\right)^{*}\right)\right] \\
& I_{y}^{\text {OUT }}=\left|E_{y}^{\mathrm{OUT}}\right|^{2}=\frac{1}{2}\left[\left|E_{x}^{\mathrm{IN}}\right|^{2}+\left|E_{y}^{\mathrm{IN}}\right|^{2}+2 \operatorname{Re}\left(i E_{x}^{\mathrm{IN}} E_{y}^{\mathrm{IN} *}\right)\right]
\end{aligned}
$$

so that the signal retrieved by the balanced detector is the following:

$$
\mathrm{S}_{\mathrm{QWP}}=I_{x}^{\mathrm{OUT}}-I_{y}^{\mathrm{OUT}}=-2 \operatorname{Im}\left(E_{x}^{\mathrm{IN}} E_{y}^{\mathrm{IN} *}\right)
$$

In the case of BD-RIKE, one has $E_{X}=E_{S}, E_{Y}=\Delta E_{S}$, so that the balanced detector output is the following:

$$
\begin{aligned}
& S_{\mathrm{HD}}=2 \operatorname{Re}\left(E_{S} \Delta E_{S}^{*}\right) \\
& S_{\mathrm{QD}}=2 \operatorname{Im}\left(E_{S} \Delta E_{S}^{*}\right)
\end{aligned}
$$

Equations (22a) and (22b) highlight the strength of BD-RIKE:

1) analogously to SRS, it provides self-heterodyne amplification of the nonlinear signal $\Delta E_{S}$, multiplying it by the strong and phase-locked Stokes field $E_{S}$;

2) differently from SRS, where the signal is superimposed on the large Stokes background $\left|E_{S}\right|^{2}$ (Eqn (7)), BD-RIKE is free from linear background because $S_{\mathrm{HD}}$ and $S_{\mathrm{QD}}$ are zero in the absence of Raman interaction (Eqns (22a) and (22b)). Thus, in principle, BD-RIKE would not require a modulation transfer technique to extract the nonlinear signal; it is anyway helpful to avoid spurious signals related to spatially varying static birefringence of the sample;

3) thanks to the inherent noise suppression provided by balanced detection, BD-RIKE is less affected than SRS by fluctuations of the Stokes field, which becomes particularly important to approach shot-noise limited detection conditions.

The functional dependence of the BD-RIKE signal on $\chi^{(3)}$ can be derived by introducing Eqns (8)-(9) into Eqns (22a) and (22b). Four different BD-RIKE responses can be selected depending on the excitation [half/quarter waveplate in excitation (HE/QE)] and detection [half/quarter waveplate in detection (HD/QD)] configuration used, as summarized in Table 1. In three out of four configurations (namely), the BD-RIKE signal is free from NRB, in two cases (QE/QD and HE/HD) proportional to the imaginary part and in a third case $(\mathrm{QE} / \mathrm{HD})$ to the real part of the nonlinear susceptibility. The ability to switch between imaginary and real part by simply changing the detection conditions (from QD to HD) represents a unique feature of BD-RIKE with respect to CARS and SRS, as discussed in the experimental part.

\section{Experimental setup}

The experimental setup used to test BD-RIKE is shown in Fig. 2. The laser source (for a detailed description see Kumar et al. ${ }^{[33]}$ ) is a mode-locked Er:fiber oscillator (FemtoFiber pro, from Toptica Photonics) operating at $1.55 \mu \mathrm{m}$ and equipped with two amplified outputs, each of which produces $350-\mathrm{mW}, 60-\mathrm{fs}$ pulses at $40 \mathrm{MHz}$ repetition rate. The first output, after frequency doubling and spectral compression in a periodically poled lithium niobate crystal, ${ }^{[34-36]}$ provides $780-\mathrm{nm} \approx 1$-ps long pulses with nearly $120 \mathrm{~mW}$ average power, which are used as a pump of the coherent Raman processes. The second output is coupled to a highly nonlinear fiber producing a supercontinuum whose spectral extension can be easily tailored by control of the chirp of the pulses coupled to the fiber. In particular, the Raman solitons generated within the supercontinuum can be tuned from 1.7 to $2.1 \mu \mathrm{m}$, so as to generate, after frequency doubling and spectral compression in a fan-out periodically poled lithium niobate crystal, tunable Stokes pulses in the range from 0.85 to $1.05 \mu \mathrm{m}$, with an average power varying from 4 to $10 \mathrm{~mW}$, depending on the 
Table 1. Scheme of the different excitation/detection configurations in BD-RIKE. The dashed yellow arrows indicate the polarization state of the Stokes field in the presence of a RIKE signal.

\begin{tabular}{|c|c|c|c|c|}
\hline $\begin{array}{c}\text { Configu- } \\
\text { ration }\end{array}$ & $\begin{array}{l}\text { Stokes } \\
\text { IN }\end{array}$ & $\begin{array}{l}\text { Pump IN } \\
\text { (after } \\
\text { WP1) } \\
\end{array}$ & $\begin{array}{l}\text { Stokes } \\
\text { (after } \\
\text { WP2) }\end{array}$ & BD-RIKE Response \\
\hline QE-QD & & & & $\operatorname{Im}\left[\chi_{1122 R}^{(3)}-\chi_{1212 R}^{(3)}\right]$ \\
\hline QE-HD & & & & $\operatorname{Re}\left[\chi_{1122 R}^{(3)}-\chi_{1212 R}^{(3)}\right]$ \\
\hline HE-QD & & & & $\operatorname{Re}\left[\begin{array}{l}\chi_{1122 R}^{(3)}+\chi_{1212 R}^{(3)}+ \\
\chi_{1122 N R}^{(3)}+\chi_{1212 N R}^{(3)}\end{array}\right]$ \\
\hline HE-HD & & & & $\operatorname{Im}\left[\chi_{1122 R}^{(3)}+\chi_{1212 R}^{(3)}\right]$ \\
\hline
\end{tabular}

BD-RIKE, balanced-detection Raman-induced Kerr effect. HE/QE/HD/QD, half/quarter waveplate in excitation/detection. This table is available in colour online at wileyonlinelibrary.com/journal/jrs.

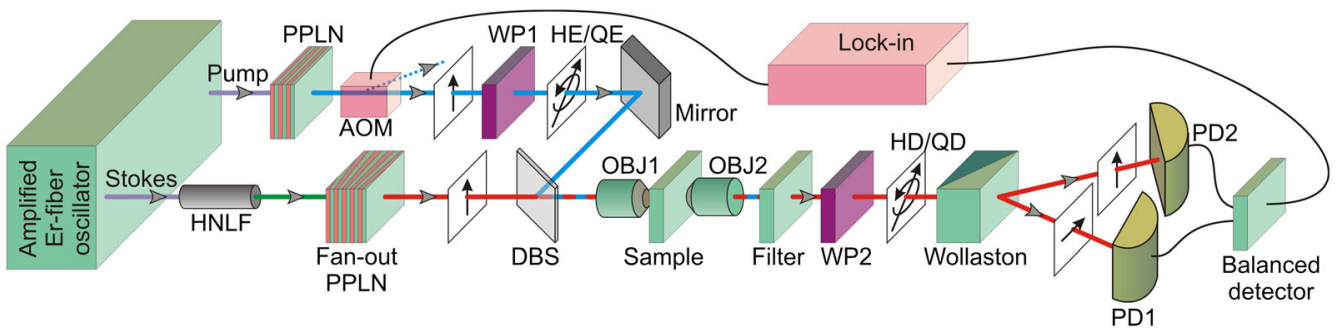

Figure 2. Scheme of the experimental setup: HNLF, highly nonlinear fiber; PPLN, periodically poled lithium niobate; AOM, acousto-optical modulator; WP, waveplates; DBS, dichroic beam splitter; HE/QE/HD/QD, half/quarter waveplate in excitation/detection, as described in the main text; OBJ, objectives; PD, photodiodes.

wavelength. In this way, it is possible to cover with the pumpStokes detuning a wide range of biologically relevant frequencies, from 1000 to $3500 \mathrm{~cm}^{-1}$.

The pump beam path is aligned along the zeroth diffraction order of an acousto-optic modulator driven at $70 \mathrm{kHz}$. A birefringent waveplate assigns the desired polarization state to the pump, which is either linear at $45^{\circ}$ with respect to the Stokes or circular, according to the HE or QE excitation modes, respectively. After synchronization by a delay line, pump and Stokes pulses are collinearly combined by a dichroic beam splitter and focused by a microscope objective onto the sample. The Stokes pulse is then selected by a sequence of long-pass filters and sent to a detection chain composed of a waveplate, Wollaston prism, and a pair of identical InGaAs photodiodes, followed by a differential amplifier, the output of which is sent to a lock-in amplifier. The waveplate on detection, which is a QWP (HWP) for the HD (QD) detection mode, alters the polarization state of the Stokes beam incident on the Wollaston prism so as to extract a zero signal out of the differential amplifier in pump-off conditions.

\section{Results and discussion}

Balanced-detection RIKE was first tested in the spectroscopic mode, by measuring the spectral response of a sample of methanol as a function of the four possible excitation/detection conditions. Figure 3 shows the spectra obtained in the different cases when tuning the Stokes wavelength in the spectral range encompassing the $\mathrm{CH}$ vibrational modes. The results are in agreement with the predictions of Table 1: in two cases (QE-QD and HE-HD configurations), the spectrum exhibits the bell-shaped response characteristic of the $\operatorname{Im}\left[\chi^{(3)}\right]$ resonant vibrational response, whereas in the other two cases (QE-HD and HE-QD configurations), the spectrum exhibits the typical dispersive behavior around the resonance given by the $\operatorname{Re}\left[\chi^{(3)}\right]$ component. A small deviation only occurs for the QE-QD configuration, because of the nonperfect phase retardation introduced by the QWP over the whole tuning range. In three out of four configurations (with the exception of the HE-QD configuration), the NRB is efficiently rejected, again in excellent agreement with theory.

Figure 4 reports a quantitative comparison of the signals provided by CARS, SRS, and BD-RIKE in the case of a methanol sample and under the same experimental conditions. As expected, methanol resonances appear sharper in CARS because of the quadratic dependence on $\chi^{(3)}$, and also slightly shifted because of the interplay with the NRB. On the contrary, SRS and BD-RIKE (here detected in the HE-HD configuration) provide nearly identical responses, closely reproducing the spontaneous Raman spectrum of methanol. However, the vertical scale highlights big differences between the signal intensities in the three cases: the CARS signal is at the 10-pW level, i.e. nearly four orders of magnitude lower than SRS and BD-RIKE, which undergo selfheterodyne amplification through the $\mathrm{mW}$-level Stokes field, according to Eqns (7), (22a), and (22b), respectively. On the other hand, the SRS signal sits on a large linear background given by the Stokes field itself. This does not occur in the BD-RIKE signal 


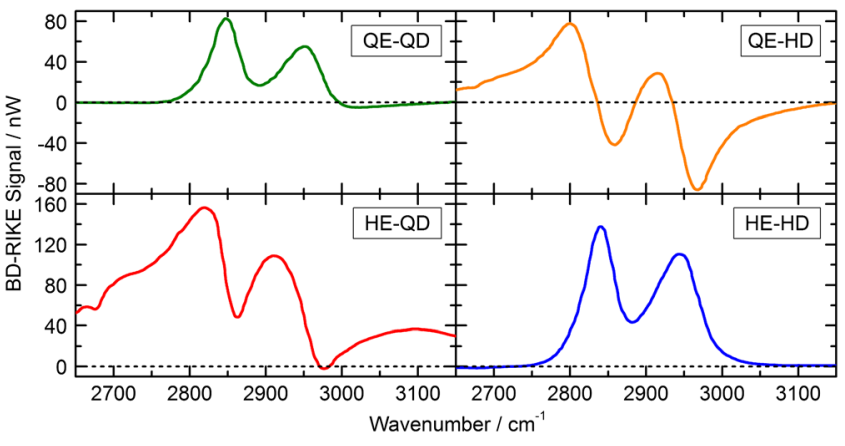

Figure 3. Balanced-detection Raman-induced Kerr effect (BD-RIKE) signal of pure methanol measured using the four configurations in excitation/ detection. HE/QE/HD/QD, half/quarter waveplate in excitation/detection.

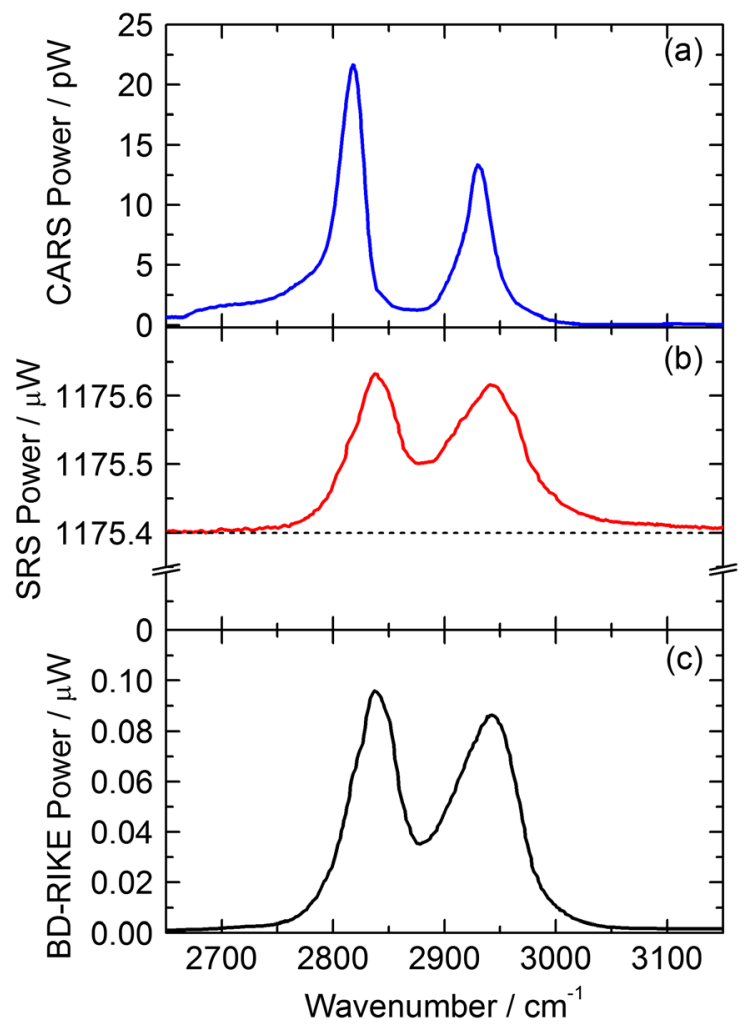

Figure 4. Quantitative comparison of the signals provided by (a) coherent anti-Strokes Raman scattering (CARS), (b) stimulated Raman scattering (SRS), and (c) balanced-detection Raman-induced Kerr effect (BD-RIKE) in the same experimental conditions for a pure methanol sample.

which, thanks to the balanced detection configuration, sits on a zero background. The balanced detection mode carries another important advantage with respect to SRS, which is the rejection of the laser intensity noise. This is well highlighted in Fig. 5, where SRS and BD-RIKE signals from methanol at the peak of one of the two resonances $\left(2840 \mathrm{~cm}^{-1}\right.$ detuning) are normalized and compared with each other upon scanning the temporal delay between pump and Stokes pulses. In both cases, the temporal shape of the two curves reflects the cross-correlation between pump and Stokes pulse intensity profiles, but the important difference resides in the level of the rms noise, which is higher in the case of SRS by a factor of nearly six, as also evidenced in the inset.

To demonstrate the linear concentration dependence of BD-RIKE and its capability to detect less abundant species, we performed a

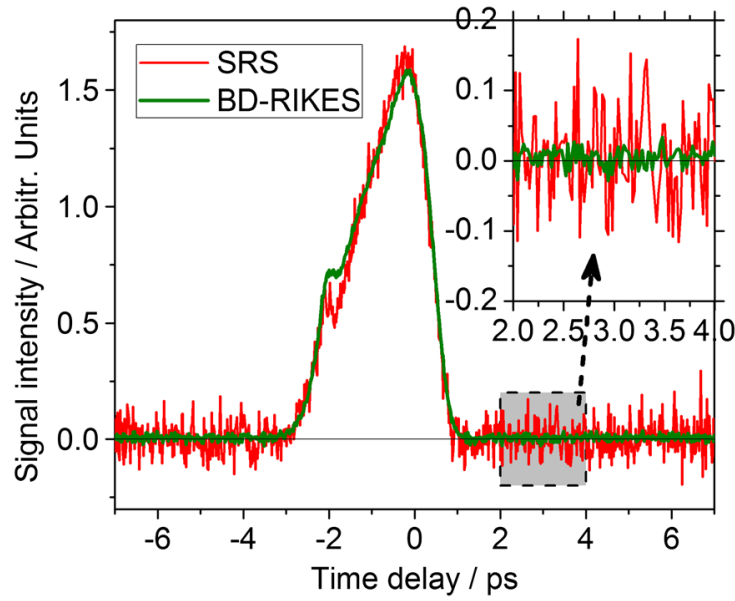

Figure 5. Direct comparison of the signal-to-noise ratio achievable using stimulated Raman scattering (SRS) and balanced-detection Ramaninduced Kerr effect (BD-RIKE) techniques. The response of a pure methanol sample has been acquired under the same experimental conditions as a function of the temporal delay between pump and Stokes pulses. Inset: zoom of the noise level in a region where no signal is present (pump/Stokes pulse delay larger than their duration). This figure is available in colour online at wileyonlinelibrary.com/journal/jrs.

dilution test of methanol in water, the results of which are reported in Fig. 6. In this case, BD-RIKE is compared with CARS only, because no appreciable differences can be found with respect to SRS. The two panels show the Raman spectra for decreasing methanol concentrations. Because of the quadratic dependence of CARS on the nonlinear susceptibility and its interplay with the NRB (Eqn 5), the CARS signal drops very quickly with the concentration and can hardly be distinguished from the water response at dilution factors of only $6 \%$. On the other hand, the Raman features of methanol are well preserved by BD-RIKE, even for dilution factors at the $1 \%$ level. It is important to note that this value does not represent the lowest detection limit, but rather the value at which the nonlinear Raman signal from water becomes comparable with that from methanol. By properly subtracting the water background or choosing a detection window in which the spectral overlap between solvent and solute is lower, even higher dilution factors would become accessible.

Coherenet Raman scattering microscopy is typically used for high-speed imaging in the narrowband mode, i.e. by acquiring the vibrational response at a single color. Although this approach is very powerful for spectrally well-separated chemical species, in the case of complex heterogeneous systems, it becomes sometimes difficult to distinguish the different components. BD-RIKE, by the combination of the QE-QD and QE-HD modes, allows to reconstruct the full vibrational response, including the vibrational phase. The additional phase information is very powerful, ${ }^{[8,37-39]}$ as different components in a mixture might have comparable vibrational amplitude but different vibrational phases at a given frequency. This is the case, for example, when the probe frequency sits in the middle between two resonances: the vibrational responses will have comparable amplitudes but a phase shift by $\approx \pi$. The vibrational phase has been so far measured using vibrational phase contrast CARS, which is a version of interferometric CARS requiring three overlapping beams generated by a phase-coherent chain. Our BD-RIKE approach enables straightforward access to the vibrational phase in a simple two-beam setup.

To highlight the potential of BD-RIKE for vibrational phase imaging, Fig. 7(a) reports the results obtained on a blend of 3- $\mu \mathrm{m}$ 

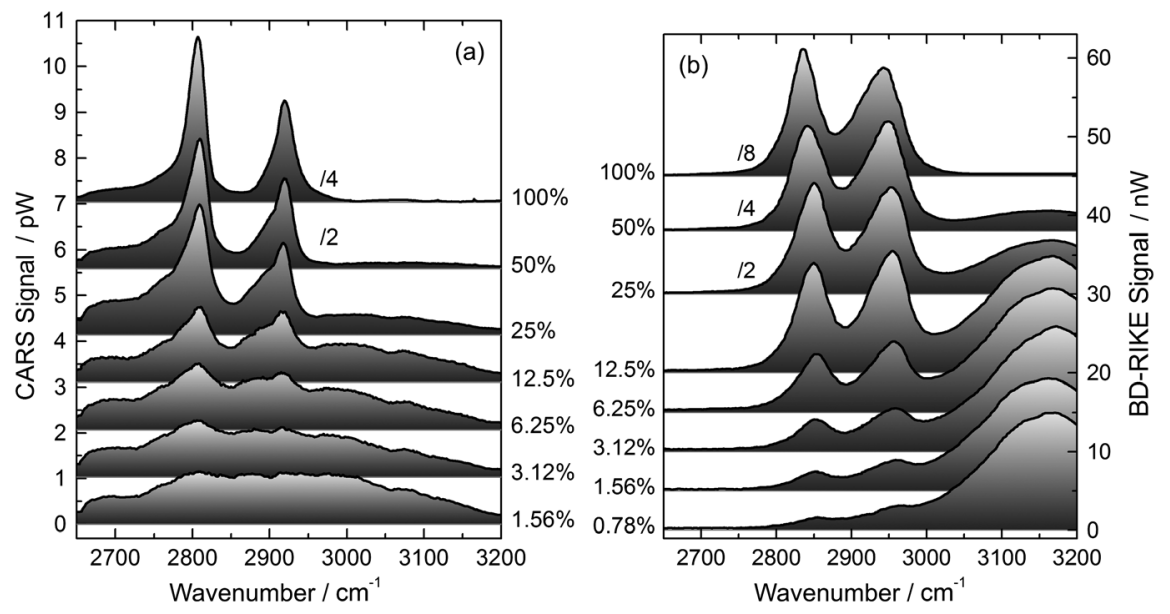

Figure 6. Dilution test of methanol in water for (a) Coherent anti-Strokes Raman scattering (CARS) and (b) balanced-detection Raman-induced Kerr effect (BD-RIKE) (under the half waveplate in excitation/detection configuration). The labels refer to the methanol concentration.
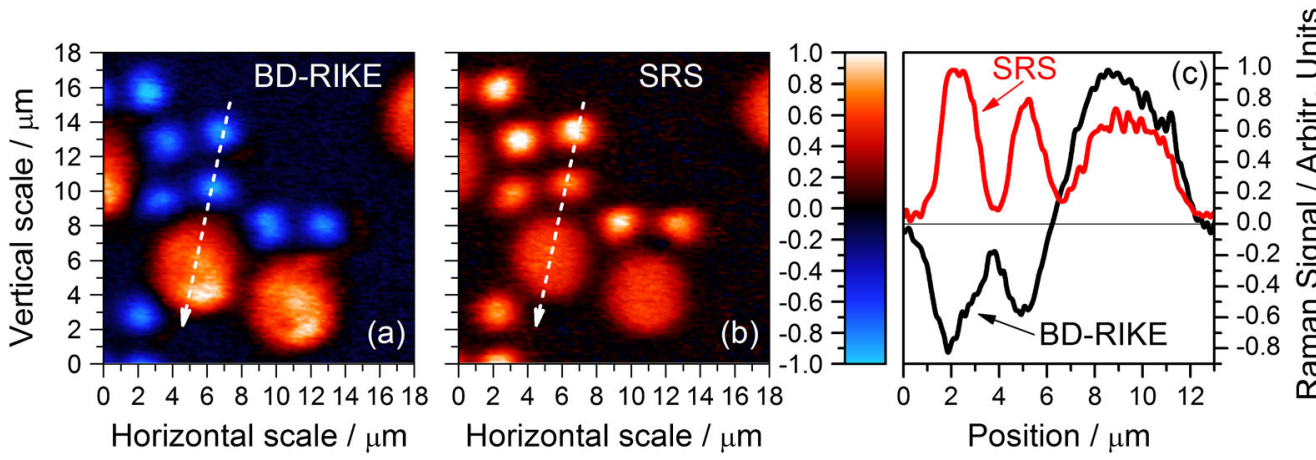

Figure 7. Microscopy image of a blend of polystyrene and polymethylmethacrylate bead blend over a $18 \times 18 \mu \mathrm{m}^{2}$ area measured at $3060 \mathrm{~cm}^{-1} \mathrm{frequency} \mathrm{in}$ the case of (a) balanced-detection Raman-induced Kerr effect (BD-RIKE) in the quarter/half waveplate in excitation and detection configuration and (b) stimulated Raman scattering (SRS). (c) Normalized intensity profiles extracted along the dashed white lines in (a) and (b), highlighting the change in sign in the BD-RIKE signal for the polystyrene (negative) and polymethylmethacrylate (positive) beads. This figure is available in colour online at wileyonlinelibrary.com/journal/jrs.

polystyrene and 6- $\mu \mathrm{m}$ polymethylmethacrylate beads. The beads have been probed using a pump/Stokes detuning of $\approx 3060 \mathrm{~cm}^{-1}$ and a QE-HD configuration, which allows the real part of the Raman response to be accessed. With such choice, polystyrene and polymethylmethacrylate beads produce signals of opposite sign. As a consequence, the two beads result to be readily distinguishable without using any two-color probing schemes, as required by SRS and CARS. This is attested in panel (b), where the SRS image acquired under the same experimental conditions does not provide any chemical selectivity. The change of sign is well highlighted in panel (c), where the intensity profiles along the dotted lines are reported for SRS and BD-RIKE.

\section{Conclusions}

In this paper, we have proposed and discussed, both theoretically and experimentally, a new coherent Raman microscopy technique, which we call BD-RIKE. This technique sensitively measures the Raman-induced polarization rotation of the Stokes pulse through a proper combination of waveplates, Wollaston prism and balanced detector that can be seamlessly integrated in existing CRS setups with minimal technological effort. BD-RIKE presents several interesting features as compared with the widely adopted CARS and SRS techniques. With respect to CARS, it measures a purely imaginary-or real-Raman response, free from NRB, and exhibits a linear concentration dependence. The advantages over SRS are more subtle but still evident, at least for samples without excessive birefringence. The balanced detection scheme allows for a higher signal-to-noise ratio when using relatively noisy lasers such as fiber lasers. Moreover, thanks to the possibility to measure both the imaginary and the real part of the nonlinear susceptibility, BD-RIKE gives direct access to the vibrational phase. This enables with single-color excitation the discrimination between different species without the need to tune the Stokes/pump wavelength as in SRS. BD-RIKE is thus believed to have great and versatile imaging capabilities and to enhance consistently the arsenal of CRS techniques.

\section{Acknowledgement}

The authors acknowledge support by the EU FP7 FET project CROSS TRAP (Contract No. ICT-244068) and by the project of RegioneLombardia (CUP No. D41J10000300007).

\section{References}

[1] C. L. Evans, X. S. Xie, Annu. Rev. Anal. Chem. 2008, 1, 883.

[2] T. Hellerer, C. Axäng, C. Brackmann, P. Hillertz, M. Pilon, A. Enejder, Proc. Natl. Acad. Sci. U.S.A. 2007, 104, 14658.

[3] G. L. Eesley, J. Quant. Spectrosc. Radiat. Transfer 1979, 22, 507. 
[4] G. Turrell, J. Corset, Raman Microscopy: Developments and Applications, Academic Press, San Diego, 1996.

[5] A. Zumbusch, G. R. Holtom, X. S. Xie, Phys. Rev. Lett. 1999, 82, 4142.

[6] C. L. Evans, E. O. Potma, M. Puoris'haag, D. Cote, C. P. Lin, X. S. Xie, Proc. Natl. Acad. Sci. U.S.A. 2005, 102, 16807.

[7] E. O. Potma, C. L. Evans, X. S. Xie, Opt. Lett. 2006, 31, 241.

[8] M. Jurna, J. P. Korterik, C. Otto, J. L. Herek, H. L. Offerhaus, Phys. Rev. Lett. 2009, 103, 043905.

[9] F. Ganikhanov, C. L. Evans, B. G. Saar, X. S. Xie, Opt. Lett. 2006, $31,1872$.

[10] J. X. Cheng, L. D. Book, X. S. Xie, Opt. Lett. 2001, 26, 1341.

[11] A. Volkmer, L. D. Book, X. S. Xie, Appl. Phys. Lett. 2002, 80, 1505.

[12] D. Pestov, R. K. Murawski, G. O. Ariunbold, X. Wang, M. C. Zhi, A. V. Sokolov, V. A. Sautenkov, Y. V. Rostovtsev, A. Dogariu, Y. Huang, M. O. Scully, Science 2007, 316, 265.

[13] R. Selm, M. Winterhalder, A. Zumbusch, G. Krauß, T. Hanke, A. Sell, A. Leitenstorfer, Opt. Lett. 2010, 35, 3282.

[14] C. W. Freudiger, W. Min, B. G. Saar, S. Lu, G. R. Holtom, C. He, J. C. Tsai, J. X. Kang, X. S. Xie, Science 2008, 322, 1857.

[15] P. Nandakumar, A. Kovalev, A Volkmer, New J. Phys. 2009, 11, 033026.

[16] D. Fu, F. K. Lu, X. Zhang, C. Freudiger, D. R. Pernik, G. Holtom, X. S. Xie, J. Am. Chem. Soc. 2012, 134, 3623.

[17] Y. Ozeki, Y. Kitagawa, K. Sumimura, N. Nishizawa, W. Umemura, S. Kajiyama, K. Fukui, K. Itoh, Opt. Express 2010,18, 13708.

[18] B. G. Saar, C. W. Freudiger, J. Reichman, C. M. Stanley, G. R. Holtom, X. S. Xie, Science 2010, 330, 1368.

[19] E. O. Potma, D. J. Jones, J.-X. Cheng, X. S. Xie, and J. Ye, Opt. Lett. 2002, 27, 1163

[20] F. Ganikhanov, S. Carrasco, X. S. Xie, M. Katz, W. Seitz, D. Kopf, Opt. Lett. 2006, 31, 1292.

[21] S. Lefrancois, D. Fu, G. Holtom, L. Kong, W. Wadsworth, P. Schneider, R. Herda, A. Zach, X. S. Xie, F. Wise, Opt. Lett. 2012, 37, 1652.
[22] K. Nose, Y. Ozeki, T. Kishi, K. Sumimura, N. Nishizawa, K. Fukui, Y. Kanematsu, K. Itoh, Opt. Express 2012, 20, 13958.

[23] A. Gambetta, V. Kumar, G. Grancini, D. Polli, R. Ramponi, G. Cerullo, M. Marangoni, Opt. Lett. 2010, 35, 226.

[24] J. P. Pezacki, J. A. Blake, D. C. Danielson, D. C. Kennedy, R. K. Lyn, R. Singaravelu, Nature Chem. Biol. 2011, 7, 137.

[25] D. Heiman, R. W. Hellwarth, M. D. Levenson, G. Martin, Phys. Rev. Lett. 1976, 36, 189

[26] S. Shim, R. A. Mathies, J. Raman Spectrosc. 2008, 39, 1526.

[27] B. R. Bachler, M. E. Fermann, J. P. Ogilvie, Opt. Express 2012, 20, 835.

[28] C. W. Freudiger, M. B. J. Roeffaers, X. Zhang, B. G. Saar, W. Min, X. S. Xie, J. Phys. Chem. B 2011, 115, 5574.

[29] Q. Wu, X.-C. Zhang, Appl. Phys. Lett. 1996, 68, 1604.

[30] Q. Wu, X.-C. Zhang, Appl. Phys. Lett. 1997, 71, 1285.

[31] M. D. Levenson, S. S. Kano, Introduction to Nonlinear Laser Spectroscopy, Academic Press, San Diego, 1988.

[32] E. Hecht, Optics, 4th edition, Pearson/Addison-Wesley, San Francisco, 2002.

[33] V. Kumar, M. Casella, E. Molotokaite, D. Polli, G. Cerullo, and M. Marangoni, J. Raman Spectrosc. 2012, 43, 662.

[34] M. Marangoni, D. Brida, M. Quintavalle, G. Cirmi, F. M. Pigozzo, C. Manzoni, F. Baronio, A. D. Capobianco, G. Cerullo, Opt. Express 2007, 15, 8884.

[35] M. Marangoni, A. Gambetta, C. Manzoni, V. Kumar, R. Ramponi, G. Cerullo, Opt. Lett. 2009, 34, 3262.

[36] G. Krauss, T. Hanke, A. Sell, D. Träutlein, A. Leitenstorfer, R. Selm, M. Winterhalder, A. Zumbusch, Opt. Lett. 2009, 34, 2847.

[37] M. Jurna, E. T. Garbacik, J. P. Korterik, J. L. Herek, C. Otto, H. L. Offerhaus, Anal. Chem. 2010, 82, 7656.

[38] H. L. Offerhaus, E. T. Garbacik, A. C. W. van Rhijn, A. L. Fussell, J. L. Herek, Rev. Anal. Chem. 2012, 31, 1.

[39] A. Wipfler, J. Rehbinder, T. Buckup, M. Motzkus, Opt. Lett. 2012, 37, 4239. 